Research Article

\title{
Multiobjective Optimization of Safety Risk of Prefabricated Building Construction considering Risk Correlation
}

\author{
Chunguang Chang, ${ }^{1}$ Xi Wu $\mathbb{D}^{1},{ }^{1}$ and Xin Yan $^{2}$ \\ ${ }^{1}$ School of Management, Shenyang Jianzhu University, Shenyang 110168, China \\ ${ }^{2}$ School of Statistics and Management, Shanghai University of Finance and Economics, Shanghai 200433, China \\ Correspondence should be addressed to Xi Wu; wuxi2020sjzu@163.com
}

Received 1 November 2019; Revised 16 March 2020; Accepted 4 April 2020; Published 27 April 2020

Academic Editor: Alessandro Contento

Copyright ( $) 2020$ Chunguang Chang et al. This is an open access article distributed under the Creative Commons Attribution License, which permits unrestricted use, distribution, and reproduction in any medium, provided the original work is properly cited.

\begin{abstract}
The analysis of the overall risk level of the system and the reduction of risk control costs are the key links to avoid the safety risks of prefabricated construction. In this paper, a dual-objective optimization model is constructed with the objective of minimizing system security risk loss and control cost to minimize the level of construction safety risks for prefabricated building while minimizing cost consumption. This paper introduces risk correlation into the optimization model based on the 2-additive fuzzy measure and solves it with discrete multiobjective particle swarm optimization (discrete-MOPSO). The example shows that considering the correlation of risks in different situations can better reflect the interaction relationship between the risk factors of building construction safety and improve the rationality of the optimization model.
\end{abstract}

\section{Introduction}

Although the effective risk control method is requisite for successful risk management in industrial construction projects, the risk managing and controlling skills of managers at various levels may not conform to the requirements of new construction management systems [1]. Effective control of multiple risk factors of prefabricated buildings, which are a major form of industrialized buildings, needs reasonable adoption of coping measures. Upon the occurrence of a risk, managers must mobilize relevant resources to manage the emergency. At this time, neither the resource utilization efficiency nor the risk management effect is easily optimizable. Hence, controlling the risk level within an acceptable range through efficient risk optimization modeling is a hot topic in the research of building construction safety at present.

Given the complex environment of prefabricated construction sites and the limited manpower and capital resources involved in risk management, the control measures adopted for different hazard sources vary. Accordingly, the required control costs vary, and the contributions to overall system risk levels also vary. This introduces the multiobjective optimization problem of prefabricated buildings. When handling multiobjective optimization problems, their noninferior solution sets are sought generally to make multiobjective decisions based on the Pareto frontier distribution since these problems often do not have a unique optimal solution. With the development of prefabricated buildings, which is a novel building system, the relevant risk factors have been increasing. Various risk factors interact with each other to result in more complicated relationships between risk events and risk factors. Since the relationships between certain risk factors may lead to risk events, the relational influences between correlated risks can be considered in the optimization model analysis to better rationalize the risk optimization control [2].

Respecting the multiobjective optimization control, a variety of modified multiobjective particle swarm optimization (MOPSO) algorithms have been proposed by many scholars after the algorithmic proposal by Coello Coello and Lechuga [3], which have been applied in multiple fields $[4,5]$. 
Regarding risk correlation, Li et al. [6] analyzed the risk relevance of prefabricated buildings from the perspective of the association between risks and various stakeholders. They put forward an effective method for analyzing stakeholderrelated risk factors and assessed the impacts of these risk factors from a network perspective. Based on the metanetwork analysis, Wang et al. [7] developed an effective way to express the complex interactions among various factors involved in a project and extended the analytical scope to multiple dimensions to form an integrated project network covering relationships between various influencing factors. By employing the system dynamics model and Monte Carlo simulation, Li et al. [8] modeled and simulated the influences of various risks on the progress of prefabricated construction, thereby identifying the potential impacts of various risk types on the scheduling of prefabricated construction projects. Tavakolan and Etemadinia [9] confirmed the importance of interactions and fuzzy numbers between risks through the fuzzy weighted interpretive structural modeling (FWISM). They proposed a risk interaction network for construction projects, which offered necessary means for exploring the influence and dependence among risk factors. Concerning the research of prefabricated buildings, Kim et al. [10] proposed a supply chain cost model using timedriven activity-based costing for the cost minimization problem of multiskilled resources in the prefabricated buildings. Mehrdad et al. [11] incorporated the cost and time required for cross-training multiskilled resources in fabricated buildings into the resource planning calculation, which used the integer and probability optimization models to minimize the cost of utilizing multiskill resources in offsite construction. To optimize the cost management of prefabricated buildings and lower the capital cost, Xue et al. [12] explored the variables affecting high prefabrication capital cost and optimized the cost by developing FAEM. Yang et al. [13] created an inventory management model for building materials, which combined the construction engineering characteristics with the order strategy, and achieved the optimal inventory cost of building materials by solving the genetic algorithm.

Although the above findings have enriched the theoretical methods concerning risk relevance and risk cost optimization in prefabricated buildings, none of the previous studies has taken into account the potential impacts of the correlation between various risk factors on the risk control costs and expected losses. Hence, this paper builds a safety risk optimization model for prefabricated building construction from a risk correlation perspective and solves the model using the MOPSO algorithm.

\section{Problem Description}

The goals of correlation analysis are to figure out the interrelations between different risks and to investigate whether the correlations between various risk factors affect the subject of research. Prefabricated construction involves numerous risk factors, which are intricately related and mutually influential. For instance, builders may operate illegally during the construction, and some types of machinery and equipment may not be inspected or maintained regularly. Casualties are possible when these two risks occur concurrently, thus increasing the costs of project coordination and accident compensation. Consideration of correlations among different risks is the focus of this paper since ignoring the inter-risk correlations will lead to an overly idealistic strategy for risk control. The implementation process of prefabricated building projects will be affected by various external risk factors. Under limited resources, risk managers are more willing to take targeted measures in order to attain optimal allocation of resources and improved efficiency of risk management. To minimize the construction safety risk level of prefabricated building projects while lowering the cost consumption as far as possible, this paper builds a biobjective optimization model to balance the system risk level and reduce the cost of risks by taking the minimization of system safety risk loss and risk control cost as the objective functions. Constraints set in the model satisfy the following requirements: the safety risk level of prefabricated construction shall not exceed the maximum permissible value of the entire system, and the risk control cost shall not exceed the cost ceiling set for risk control optimization.

\section{Risk Optimization Modeling for Prefabricated Construction}

\subsection{Symbol and Variable Description}

$n$ : number of risk sources in risk control optimization

$R$ : safety risk set for prefabricated construction

$R_{i}: i$-th risk source

$P_{i}$ : probability of $i$-th risk occurrence

$L_{i}$ : loss caused by the $i$-th risk

$x_{i}$ : decision variable of the $i$-th risk source; $x_{i}=1$ indicates that the $i$-th risk source is controlled, whereas $x_{i}=0$ indicates that the $i$-th risk source is not controlled

$\lambda_{i}$ : weight coefficient of the $i$-th risk source

$V\left(R_{i}\right)$ : expected loss from the $i$-th risk

$V(R)$ : expected loss from the overall system risk

$V(R)_{\lambda}$ : expected loss from the overall system risk with magnified effect

$V(R)^{\prime}$ : expected loss from the overall system risk when considering the risk loss correlation

$V(R)_{\lambda}^{\prime}$ : expected loss from the overall system risk with magnified effect when considering the risk loss correlation

$V(R)_{\max }$ : maximum permissible value of expected loss from the overall system risk

$C\left(R_{i}\right)$ : cost of optimally controlling the $i$-th risk source $C(R)_{\max }$ : cost ceiling set for the risk control optimization

$L_{i j}$ : loss caused by concurrent occurrence of $i$-th risk $R_{i}$ and $j$-th risk $R_{j}$ 
$P_{i j}$ : probability of concurrent occurrence of $i$-th risk $R_{i}$ and $j$-th risk $R_{j}$

$\mu$ : fuzzy measure

$I_{i j}$ : interaction coefficient between losses $L_{i}, L_{j}$ from risk sources $R_{i}, R_{j}$, with a value range of $[-1,1]$

\subsection{Basic Model of Prefabricated Building Risk Optimization.} In constructional engineering, risk can be described as an integration of probabilities and impact outcomes that have potentially adverse impacts on the people's life and property safety or projects. Generally, risk has the following two characteristics: uncertainty and hazard. The risk set of prefabricated construction projects is denoted by $R=\left\{R_{i} ; i=1,2, \ldots, n\right\}$. In this paper, the fundamental formula of risk measurement is used to define the expected loss from the $i$-th risk as

$$
V\left(R_{i}\right)=\left(1-x_{i}\right) P_{i} L_{i}, \quad i=1,2, \ldots, n,
$$

where $1-x_{i}=0$ if $x_{i}=1$. In other words, the expected loss from the $i$-th risk in formula (1) is zero.

When the risk correlation is not considered, the expected loss $V(R)$ from the overall system risk is equal to the sum of expected losses from various risks, that is,

$$
V(R)=\sum_{i=1}^{n}\left(1-x_{i}\right) V\left(R_{i}\right)=\sum_{i=1}^{n}\left(1-x_{i}\right) P_{i} L_{i} .
$$

The total risk control cost is the sum of costs for controlling various risks, that is,

$$
C(R)=\sum_{i=1}^{n} x_{i} C\left(R_{i}\right)
$$

For conservative consideration, this paper appropriately magnifies the loss caused by risk occurrence on the basis of general loss value $L_{i}$. Meanwhile, the varying importance levels of different factors to the entire system are taken into account. Accordingly, the loss magnification coefficient $e^{\lambda_{i}}$ for risk source $R_{i}$ is set based on the magnification effect combined with the importance degree. Hence, the expected loss from the overall system risk with magnification effect without considering the risk correlation is

$$
V(R)_{\lambda}=\sum_{i=1}^{n} e^{\lambda_{i}}\left(1-x_{i}\right) P_{i} L_{i} .
$$

Such a risk optimization model can be expressed as

$$
\begin{aligned}
& \min V(R)_{\lambda}, \\
& \min C(R), \\
& \text { s.t. } V(R)_{\lambda} \leq V(R)_{\max }, \\
& C(R) \leq C(R)_{\max }, \\
& x_{i} \in\{0,1\}, \quad i=1,2, \ldots, n,
\end{aligned}
$$

where formula (5) expresses that the expected system risk loss is the minimum; formula (6) expresses that the input cost is the minimum; formula (7) expresses that the expected system risk loss shall not exceed the maximum permissible value; and formula (8) expresses that the risk control cost shall not exceed the cost ceiling set for the risk control and optimization.

3.3. Risk Optimization Model considering Risk Correlation. It is clear from the definition of risk that risk involves both risk occurrence probability and risk loss. Thus, inter-risk correlations can be summarized from these two aspects. Since one optimization goal of our proposed model is expected risk loss, the correlations between risks are depicted mainly from the risk loss dimension. The loss effect resulting from each risk is not simply superimposed. Loss generated by concurrent occurrence of two risks may be greater than, equal to, or less than the sum of losses caused when the two risks occur independently. Their magnitude relationship can be expressed in three scenarios:

(1) $L_{i j}>L_{i}+L_{j}$, which indicates that the loss resulting from concurrent occurrence of two risks $i, j$ is greater than the loss caused by their separate occurrence. The risk loss values exhibit a complementary relationship.

(2) $L_{i j}=L_{i}+L_{j}$, which indicates that the two risks $i, j$ do not influence each other at the time of occurrence. The risk loss values present an independent and superposable relationship.

(3) $L_{i j}<L_{i}+L_{j}$, which indicates that the loss resulting from concurrent occurrence of two risks $i, j$ is less than the loss caused by their separate occurrence. The risk loss values exhibit a redundant relationship.

In the existing literature, fuzzy measures are a common approach to measure the nonadditivity of losses, which are a means of expressing interaction. Accordingly, the fuzzy integral can be used for the integration of assessment data [14-16]. Common fuzzy measures include $k$-additive fuzzy measure and $g_{\lambda}$ fuzzy measure. As a type of $k$-additive fuzzy measure, the 2 -additive fuzzy measure gives equal consideration to the expressivity of measure and the complexity of the algorithm, which has thus been applied extensively in multiple areas $[17,18]$.

The 2-additive fuzzy measure can not only characterize the weight of each attribute but also the degree of association between the attribute set weight and the attributes. Using a combination of the 2-additive fuzzy measure with Choquet fuzzy integral, it is possible to better express the interaction between indices and aggregation of index weights. Thus, the correlations exist between different risk factors which are represented in this paper using the 2-additive fuzzy measure proposed by Mayag [19]. Meanwhile, the Choquet integral operator is utilized to nonlinearly integrate the loss values of various indices [20]. 
3.3.1. Correlation Description Based on the 2-Additive Fuzzy Measure. Without considering the risk loss correlation, the sum of all risk losses is

$$
L=\sum_{i=1}^{n} L_{i}
$$

Let the set be $N=\left\{L_{i}, \ldots, L_{n}\right\}$ and the fuzzy measure $\mu: N \longrightarrow[0,1]$ satisfy the following conditions:

$$
\begin{aligned}
& \mu(\phi)=0, \\
& \mu(N)=1, \\
& \forall N_{1}, N_{2} \in N, \\
& N_{1} \subset N_{2} \Longrightarrow \mu\left(N_{1}\right) \leq \mu\left(N_{2}\right) .
\end{aligned}
$$

Assuming $L_{1}, \ldots, L_{n}$ are unequal and already sorted, i.e., $L_{1}<L_{2}<\cdots<L_{n}$. Let $N_{i}=\left\{L_{i}, \ldots, L_{n}\right\}, \quad \mu_{i}=\mu\left(N_{i}\right)$, $\Delta \mu_{i}=\mu_{i}-\mu_{i+1}$, and $L_{0}=0, \mu_{n+1}=0$. Then, the sum of all risk losses $L \prime$, after considering the risk loss correlation based on the fuzzy measure, is

$$
\begin{aligned}
L^{\prime} & =n \sum_{i=1}^{n} \mu_{i}\left(L_{i}-L_{i-1}\right) \\
& =n\left[\left(\mu_{1}-\mu_{2}\right) L_{1}+\cdots+\mu_{n} L_{n}\right] \\
& =n \sum_{i=1}^{n} \Delta \mu_{i} L_{i} .
\end{aligned}
$$

Furthermore, for the 2-additive fuzzy measure, there is

$$
\Delta \mu_{i}=\frac{1}{n}+\frac{1}{2} \sum_{u=1} I_{i u}-\frac{1}{2} \sum_{u<i} I_{i u},
$$

where $I_{i u}$ denotes the interaction coefficient between $L_{i}$ and $L_{u}$. Then, formula (14) can be expressed as

$$
\begin{aligned}
L^{\prime} & =n \sum_{i=1}^{n}\left[\frac{1}{n}+\frac{1}{2} \sum_{u>i} I_{i u}-\frac{1}{2} \sum_{u<i} I_{i u}\right] L_{i} \\
& =L+\frac{n}{2} \sum_{i=1}^{n}\left(\sum_{u>i} I_{i u}-\sum_{u<i} I_{i u}\right) .
\end{aligned}
$$

On this basis, correlated risk loss pairs are assumed to have no external correlation. That is, if there is correlation between $L_{i}$ and $L_{j}$, then $L_{i}$ is independent of any risk loss except for $L_{j}$, and $L_{j}$ is also independent of any risk loss except for $L_{i}$. Without loss of generality, considering that there is only one correlated pair $\left(L_{i}, L_{j}\right), i<j$ among all risk losses, then

$$
\begin{aligned}
L^{\prime} & =L+\frac{n}{2} L_{i} I_{i j}-\frac{n}{2} L_{j} I_{i j} \\
& =L+\frac{n}{2}\left(L_{i}-L_{j}\right) I_{i j} .
\end{aligned}
$$

At this time, the loss value increases by $(n / 2)\left(L_{i}-L_{j}\right) I_{i j}$ after considering the correlation between the risk loss pair as compared to the case without considering such correlation.
The loss $L_{i j}$ caused by concurrent occurrence of risks $i$ and $j$ is expressed as

$$
L_{i j}=L_{i}+L_{j}+\frac{n}{2}\left(L_{1}-L_{j}\right) I_{i j}
$$

3.3.2. Risk Optimization Modeling considering Risk Correlations. Assume that the occurrence probability between risks is independent of each other, that is, $P_{i j}=P_{i} P_{j}$. $L_{i j}-L_{i}-L_{j}$ is used to denote the elimination of risk values generated by independent occurrence of original risks, while $Z$ denotes the set of risk pairs with loss correlations. Then, the expected loss $V(R)^{\prime}$ from the overall system risk when considering the risk loss correlation is expressed as

$$
\begin{aligned}
V(R)^{\prime}= & \sum_{i=1}^{n}\left(1-x_{i}\right) P_{i} L_{i} \\
& +\sum_{i=1}^{n}\left(1-x_{i}\right)\left(1-x_{j}\right) P_{i} P_{j}\left(L_{i j}-L_{i}-L_{j}\right) .
\end{aligned}
$$

Furthermore, regarding the $L_{i j}$ magnification effect, the arithmetic mean of magnification effect coefficients of $L_{i}$ and $L_{j}$ is used after comprehensively considering the magnification effects of the two. Hence, the expected loss from the overall system risk with magnification effect when considering the risk loss correlation is

$$
\begin{aligned}
V(R)_{\lambda^{\prime}}= & \sum_{i=1}^{n} e^{\lambda_{i}}\left(1-x_{i}\right) P_{i} L_{i}+\sum_{(i, j) \in Z}\left(1-x_{i}\right)\left(1-x_{j}\right) P_{i} P_{j} . \\
& \left(\frac{e^{\lambda_{i}}+e^{\lambda_{j}}}{2} L_{i j}-e^{\lambda_{i}} L_{i}-e^{\lambda_{j}} L_{j}\right) .
\end{aligned}
$$

In this case, the optimization model considering risk correlations can be expressed as

$$
\begin{aligned}
& \min V(R)_{\lambda^{\prime}}, \\
& \min C(R), \\
& \text { s.t. } V(R)_{\lambda^{\prime}} \leq V(R)_{\max }, \\
& C(R) \leq C(R)_{\max }, \\
& x_{i} \in\{0,1\}, \quad i=1,2, \ldots, n .
\end{aligned}
$$

\section{Discrete-MOPSO Solution Design}

In the objective functions of this paper, the risk control cost and system risk loss are two mutually constrained objectives, which are a problem of combinatorial optimization. To address the problem, discrete-MOPSO is used to solve the optimization model based on the multiobjective knapsack theory. For single-objective function problems, the particle swarm optimization (PSO) finds the optimal objective solution by continuously changing the velocity and position of 
randomly initialized particle swarm through heuristic iteration and utilizes the historical and global optimal position information of particles in the course of changing velocity and position, thereby achieving closeness to the optimal solution. Regarding multiobjective function problems, there is not necessarily a consistent optimal solution. The MOPSO algorithm solves such problems by introducing the economic concept of Pareto equilibrium and performs search by efficient cluster parallel computing. The optimization results are not limited to a single-valued solution. Instead, a set of noninferior solutions can be obtained in one run. For discrete variable problems, the positional value of particles in the discrete-MOPSO algorithm is the corresponding finite or denumerable value. The problem in this paper is a discrete multiobjective problem, which aims to minimize both $V(R)$ and $C(R)$.

Let the number of particles be $m$; then, the particle position set is denoted as

$$
\mathscr{M}_{x}=\left\{\mathbf{x}_{1}, \mathbf{x}_{2}, \ldots, \mathbf{x}_{m}\right\},
$$

where $\mathbf{x}_{k}=\left(x_{k 1}, x_{k 2}, \ldots, x_{k n}\right), x_{k i} \in\{0,1\}, k=1,2, \ldots, m$; $i=1,2, \ldots, n$.

For $\forall \mathbf{x}_{h}, \mathbf{x}_{l} \in \mathscr{M}_{x}\left(\mathbf{x}_{h} \neq \mathbf{x}_{l}\right)$, its corresponding objective function values are set to $V(R)^{(h)}, C(R)^{h}$ and $V(R)^{(t)}, C(R)^{(t)}$, respectively. If $V(R)^{(h)} \leq V(R)^{l}$ and $C(R)^{(h)} \leq C(R)^{(l)}$, then the particle $\mathbf{x}_{h}$ is deemed superior to the particle $\mathbf{x}_{l}$. If $V(R)^{(h)} \leq V(R)^{l}$ and $C(R)^{(h)}>C(R)^{l}$, or $V(R)^{(h)}<V(R)^{(l)}$ and $C(R)^{(h)} \geq C(R)^{(l)}$, or $V(R)^{(h)} \geq$ $V(R)^{(l)}$ and $C(R)^{(h)}<C(R)^{(l)}$, or $V(R)^{(h)}>V(R)^{(l)}$ and $C(R)^{(h)} \leq C(R)^{(l)}$, then the particle $\mathbf{x}_{h}$ is deemed not inferior to the particle $\mathbf{x}_{l}$. A particle is regarded as a noninferior particle when it is superior or not inferior to other particles in the particle set.

The particle velocity set is denoted as

$$
\mathscr{M}_{v}=\left\{\mathbf{v}_{1}, \mathbf{v}_{2}, \ldots, \mathbf{v}_{m}\right\},
$$

where $\quad \mathbf{v}_{k}=\left(v_{k 1}, v_{k 2}, \ldots, v_{k n}\right), \quad v_{k i} \in \mathbb{R}, \quad k=1,2, \ldots, m$; $i=1,2, \ldots, n$.

Let $\varphi_{1}, \varphi_{2}$ denote the acceleration factors, $\omega_{\min }$ denote the minimum inertia weight, $\omega_{\max }$ denote the maximum inertia weight, $t$ represent the iteration round, $t=1,2, \ldots, T, k$ represent the particle serial number, $k=1,2, \ldots, m$, and $i$ represent the risk source serial number, $i=1,2, \ldots, n$. Suppose that, in the $t$-th iteration, the current position of particle $k$ is $\mathbf{x}_{k}^{(t)}$, the historical optimal position is $\mathbf{b}_{k}^{(t)}$, and the global optimal position is $\mathbf{g}^{(t)}$.

The formula for inertia weight value is updated by adopting the linearly decreasing weight (LDW) strategy proposed by Shi [21] as

$$
\omega^{(t)}=\omega_{\max }-\frac{\omega_{\max }-\omega_{\min }}{T} \cdot t .
$$

The updating formula for particle velocity is

$$
\begin{array}{r}
v_{k i}^{(t+1)}=\omega^{(t)} \cdot v_{k i}^{(t)}+\varphi_{1}, r_{k i 1}^{(t)} \cdot\left(b_{k i}^{(t)}-x_{k i}^{(t)}\right)+\varphi_{2} \cdot r_{k i 2}^{(t)} \cdot\left(g_{i}^{(t)}-x_{k i}^{(t)}\right), \\
k=1,2, \ldots, m ; i=1,2, \ldots, n,
\end{array}
$$

where $r_{k i 1}^{(t)}, r_{k i 2}^{(t)}$ is the random number respecting the independent identical distribution of Uniform $(0,1)$.

The updating formula for particle position is derived by converting velocity based on the $S$-shape-constraint transfer function $\operatorname{sigmoid}(v)$ as

$$
\begin{gathered}
\operatorname{sigmoid}\left(v_{k i}^{(t+1)}\right)=\frac{1}{1+\exp \left(-v_{k i}^{(t+1)}\right)}, \\
k=1,2, \ldots, m ; i=1,2, \ldots, n, \\
x_{k i}^{(t+1)}= \begin{cases}1, & r_{k i}^{(1+1)}<\operatorname{sigmoid}\left(v_{k i}^{(t+1)}\right) ; \\
0, & \text { others, } \\
& k=1,2, \ldots, m ; i=1,2, \ldots, n,\end{cases}
\end{gathered}
$$

where $r_{k i}^{(t+1)}$ is the random number obeying the Uniform $(0,1)$ distribution.

Step 1: determination of parameters. The number of particles $m$ is determined, as well as the iterative times $T$, the upper limit for the number of noninferior solution set elements $S_{\max }$, the inertia weight parameters $\omega_{\max }, \omega_{\min }$ in formula (28), and the acceleration factors $\varphi_{1}, \varphi_{2}$ in formula (29).

Step 2: initialization of the particle swarm position $\mathscr{M}_{x}$ and velocity $\mathscr{M}_{v}$. Concerning position initialization, the equiprobability of various position dimensions for each particle is randomized as either 0 or 1 . As for the velocity initialization, random number from the $\operatorname{Uniform}(-1,1)$ distribution is assigned as the velocity dimensions of each particle.

Step 3: the position of the current particle swarm $\left\{\mathbf{x}_{1}, \mathbf{x}_{2}, \ldots, \mathbf{x}_{m}\right\}$ is regarded as the historical optimal position $\left\{\mathbf{b}_{1}, \mathbf{b}_{2}, \ldots, \mathbf{b}_{m}\right\}$ of the particle swarm, based on which the noninferior particle set $S=\left\{\mathbf{o}_{1}, \mathbf{o}_{2}, \ldots, \mathbf{o}_{l}\right\}$ is selected.

Step 4: updating the historical optimal position of particle swarm. For each particle $k(k=1,2, \ldots, m)$, the relationships between the objective function values $V(R)$ and $C(R)$ for its current position $\mathbf{x}_{k}$ and historical optimal position $\mathbf{b}_{\mathbf{k}}$ are compared. If $\mathbf{x}_{k}$ is superior to $\mathbf{b}_{k}$, the historical optimal position is updated to $\mathbf{x}_{k}$; if $\mathbf{b}_{k}$ is superior to $\mathbf{x}_{k}$, the historical optimal position is unchanged; and if $\mathbf{x}_{k}$ and $\mathbf{b}_{k}$ present a noninferiority relationship, the historical optimal position is updated by randomly selecting one of them.

Step 5: updating the noninferior particle set. Firstly, the temporary noninferior particle set $S \prime$ is determined according to the historical optimal position of the particle swarm, and then the new noninferior particles are identified based on a combination of $S$ I and the original noninferior particle set $S$ to update $S$.

Step 6: calculating the probability information of noninferior particles in noninferior particle set $S$. For a problem with $n$ risk sources, the number of particle position space dimensions is $n$, and the value of each position dimension is either 0 or 1 . Then, the position 
space can be represented as a set of $2^{n}$ points. Assuming the particles in the noninferior solution set cover the $h$ points $H_{1}, \ldots, H_{h}$ of the position space and there are $l_{a}$ particles $\left(\sum_{a=1}^{h} l_{a}=l\right)$ at the point $H_{a}$, then the feature probability $q_{u}$ of noninferior particle $\mathbf{o}_{u}$ is

$$
q_{u}=\frac{1}{h l_{a}} \mathbf{o}_{u} \mid \in H_{a} ; u=1, \cdots, l .
$$

If $S$ is excessive, that is, $l>S_{\max }$, then $S_{\max }$ elements are randomly selected by unequal probability sampling therefrom as the noninferior solution set, where the sampling probability is the calculation result of formula (32). Afterwards, the probability information of noninferior particles is updated according to formula (32).

Step 7: updating the global optimal position. One element is randomly extracted from the noninferior solution set $S$ by equal probability sampling as the global optimal position $g$.

Step 8: the $w$ value is updated using formula (28), the particle swarm velocity $\mathscr{M}_{v}$ is updated using formula (29), and the particle swarm position $\mathscr{M}_{x}$ is updated using formula (31).

Step 9: if the iteration is completed, the position of a particle with the smallest $V(R)+C(R)$ value is selected from the noninferior particle set $S$ as the global optimal position. Otherwise, return to Step 4.

\section{Case Analysis and Model Validation}

\subsection{Risk Factor Analysis}

5.1.1. Risk Factor Index Construction and Raw Data Acquisition. On the basis of reviewing the existing literature on the safety risks of prefabricated building construction and conducting interviews with some experts and scholars in this industry, the author surveyed the prefabricated construction project $\mathrm{PH}$ of $\mathrm{X}$ city in China. Due to the protection policy, the project's information cannot be disclosed. According to the principle of risk assessment index selection, the construction safety risk factors of the project are mainly summarized into six categories: personnel, machine, material, environment, technology, and management. These six aspects mainly involve 23 risk factors. The risk weights were obtained by referring to the literature [22]. The risk accident rates were derived by statistically processing data from similar projects $[8,23]$, while the risk control costs and losses from risks (unit: 10,000 yuan) were obtained based on the relevant information of the project between 2016 and 2018 and by asking relevant personnel, which are ultimately averaged. The specific numerical values are listed in Table 1.

5.1.2. Identification of Risk Factor Correlations. To identify risk factor pairs, analysis is made on the risk factors in Table 1 . The selection principles are as follows: if the correlation of a certain risk with another risk is stronger than that with third-party risk factors, only the two risks with the strongest risk correlation are considered as the risk pair; for various risk pairs, those exhibiting stronger correlations are taken into consideration, while those with weaker correlations are excluded. After comprehensively considering the actual situation of the project, the following seven correlated rick loss pairs are identified: $\left(L_{5}, L_{19}\right),\left(L_{6}, L_{20}\right),\left(L_{11}, L_{22}\right)$, $\left(L_{12}, L_{21}\right)$ (presenting a redundant relationship), $\left(L_{4}, L_{7}\right)$, $\left(L_{2}, L_{14}\right)$, and $\left(L_{13}, L_{16}\right)$ (presenting a complementary relationship). Table 2 lists the details.

5.2. Optimization Model Solving. Based on the case studied in this paper combined with the relevant literature [24, 25] consultation, the parameters are set as follows: $\omega_{\max }=0.9$, $\omega_{\min }=0.4, T=5000, \varphi_{1}=\varphi_{2}=1.49618$, particle population size $=100$, and external memory capacity $=200$. The optimization models with and without considering correlations are solved via $R$ programming.

To reflect the specific relationship between loss from risk and risk control cost, the control cost and overall system risk loss are assumed unconstrained on the condition of sufficient funds. Figure 1 displays the mathematical outcome. As can be seen, the numbers of noninferior solutions are 93 and 110, respectively, before and after considering the risk correlations. A nonlinear relationship is found between risk loss values and risk control costs. The overall system risk loss decreases gradually with the increasing input of risk control cost, which is the maximum (approximately 3 million yuan) when the control cost is close to 0 . All risks are almost controlled at a risk control cost of around 500,000 yuan.

In actual projects, however, it is impossible to control all risks without any cost and without restrictions due to limited funds. Instead, the risks should be controlled selectively according to the actual cost budget. In this paper, four different constraints are set. Constraint one: overall risk loss ceiling $V(R)_{\max }=1$ million yuan, and total risk control cost ceiling $C(R)_{\max }=1$ million yuan. Constraint two: $V(R)_{\max }=1$ million yuan, and $C(R)_{\max }=0.3$ million yuan. Constraint three: $V(R)_{\max }=1$ million yuan, and $C(R)_{\max }=0.35$ million yuan. And constraint four: $V(R)_{\max }=1$ million yuan, and $C(R)_{\max }=0.4$ million yuan. In Figures 2 and 3, the noninferior solution sets obtained under different constraints with and without considering correlations are presented. As can be seen from Figure 2, $V(R)-C(R)$ under different constraints before and after considering correlations all exhibit approximately linear relationships. From Figure 3, it can be seen that $V(R)-$ $C(R)$ before and after considering risk correlations differ considerably at a risk control cost $C(R)$ of 0.25 million yuan when other major conditions are unchanged. In this case, consideration of risk correlations may lower more risk losses. Such difference is rather significant when $C(R)$ is 0.3 million yuan, while insignificant when $C(R)$ is 0.35 or 0.4 million yuan. This is because with the relaxation of the control cost constraint, that is, with the increase of control cost constraint value, more risk factors can be controlled. In this way, the factors that can reflect the risk correlations 
TABLE 1: Risk factors and various data for PH prefabricated construction project.

\begin{tabular}{|c|c|c|c|c|c|}
\hline Primary index & Secondary index & $\begin{array}{c}\text { Risk } \\
\text { weight }\end{array}$ & $\begin{array}{c}\text { Risk } \\
\text { accident rate }\end{array}$ & $\begin{array}{c}\text { Risk control cost } \\
(10,000 \text { yuan })\end{array}$ & $\begin{array}{c}\text { Loss from risk } \\
(10,000 \text { yuan })\end{array}$ \\
\hline \multirow{6}{*}{$\begin{array}{l}\text { Personnel risk } \\
\text { factor }\end{array}$} & \multirow{6}{*}{$\begin{array}{c}\text { Man-machine mixed operation } R_{1} \\
\text { High-altitude operation } R_{2} \\
\text { Fatigue construction caused by high-intensity operation } R_{3} \\
\text { Illegal operation by builders } R_{4} \\
\text { Lack of professional technicians } R_{5} \\
\text { Poor quality and weak safety awareness of personnel } R_{6}\end{array}$} & 0.016 & 0.53 & 0.95 & 24.25 \\
\hline & & 0.035 & 0.69 & 3.12 & 25.60 \\
\hline & & 0.019 & 0.55 & 2.22 & 7.65 \\
\hline & & 0.053 & 0.61 & 1.90 & 12.30 \\
\hline & & 0.033 & 0.54 & 0.92 & 17.95 \\
\hline & & 0.011 & 0.43 & 1.35 & 25.80 \\
\hline \multirow{3}{*}{$\begin{array}{l}\text { Mechanical risk } \\
\text { factor }\end{array}$} & \multirow{3}{*}{$\begin{array}{c}\text { Improper operation, maintenance, and safety inspection of } \\
\text { equipment } R_{7} \\
\text { Unreasonable selection and layout of transporting, hoisting, } \\
\text { and grouting equipment } R_{8} \\
\text { Equipment failure, aging } R_{9}\end{array}$} & 0.056 & 0.48 & 1.15 & 34.45 \\
\hline & & 0.037 & 0.33 & 2.87 & 6.25 \\
\hline & & 0.074 & 0.63 & 2.82 & 12.70 \\
\hline \multirow{3}{*}{ Material risk factor } & $\begin{array}{l}\text { Unqualified safety protection items related to construction } \\
\qquad R_{10}\end{array}$ & 0.078 & 0.59 & 4.62 & 28.70 \\
\hline & $\begin{array}{l}\text { Nonstandard building materials used in the construction } \\
\qquad R_{11}\end{array}$ & 0.050 & 0.46 & 4.22 & 21.80 \\
\hline & Arbitrary stacking of construction materials $R_{12}$ & 0.039 & 0.32 & 2.12 & 9.30 \\
\hline \multirow{5}{*}{$\begin{array}{l}\text { Environmental risk } \\
\text { factor }\end{array}$} & Narrow working surface, insufficient lighting $R_{13}$ & 0.071 & 0.36 & 1.97 & 12.80 \\
\hline & Improper protection measures for borders and openings $R_{14}$ & 0.066 & 0.45 & 1.30 & 16.80 \\
\hline & $\begin{array}{l}\text { Presence of hazardous sources like charged high-voltage } \\
\text { wires and underground gas pipelines around the }\end{array}$ & 0016 & 029 & 245 & 34.90 \\
\hline & construction site $R_{15}$ & 0.01 & 0.29 & 2.4 & 34.90 \\
\hline & Awful weather $R_{16}$ & 0.014 & 0.31 & 1.80 & 18.25 \\
\hline \multirow{3}{*}{$\begin{array}{l}\text { Technological risk } \\
\text { factor }\end{array}$} & $\begin{array}{l}\text { Defects in safety protection and management technologies } \\
\text { such as temporary support system } R_{17}\end{array}$ & 0.066 & 0.58 & 2.62 & 11.30 \\
\hline & $\begin{array}{c}\text { Defects in construction technologies such as hoisting } \\
\text { equipment attachment measures } R_{18}\end{array}$ & 0.056 & 0.39 & 1.47 & 6.75 \\
\hline & $\begin{array}{l}\text { Defects in construction techniques such as prefabricated } \\
\text { component assembly and key part processing } R_{19}\end{array}$ & 0.045 & 0.64 & 0.82 & 22.95 \\
\hline \multirow{4}{*}{$\begin{array}{l}\text { Management risk } \\
\text { factor }\end{array}$} & Lack of relevant safety education or training $R_{20}$ & 0.066 & 0.54 & 1.87 & 29.80 \\
\hline & $\begin{array}{l}\text { Mismatch between the safety management system and } \\
\text { actual construction conditions } R_{21}\end{array}$ & 0.026 & 0.49 & 1.60 & 14.45 \\
\hline & $\begin{array}{c}\text { Lack of unified, effective management standards or } \\
\text { supervision mechanism } R_{22}\end{array}$ & 0.032 & 0.58 & 3.55 & 49.35 \\
\hline & $\begin{array}{l}\text { Improper multiparty coordination management during } \\
\text { construction } R_{23}\end{array}$ & 0.043 & 0.61 & 2.80 & 32.65 \\
\hline
\end{tabular}

TABLE 2: Risk pairs with risk correlations.

\begin{tabular}{|c|c|c|c|}
\hline No. & Correlated risk pair & $\begin{array}{l}\text { Loss from a single risk } \\
(10,000 \text { yuan })\end{array}$ & $\begin{array}{l}\text { Loss from concurrent risks } \\
(10,000 \text { yuan })\end{array}$ \\
\hline \multirow[b]{2}{*}{1} & Lack of professional technicians $R_{5}$ & 17.95 & \multirow[b]{2}{*}{29.40} \\
\hline & $\begin{array}{c}\text { Defects in construction techniques such as prefabricated component } \\
\text { assembly and key part processing } R_{19}\end{array}$ & 22.95 & \\
\hline \multirow[b]{2}{*}{2} & Illegal operation by builders $R_{4}$ & 12.30 & \multirow[b]{2}{*}{72.22} \\
\hline & $\begin{array}{l}\text { Improper operation, maintenance, and safety inspection of equipment } \\
\qquad R_{7}\end{array}$ & 34.45 & \\
\hline 3 & $\begin{array}{l}\text { Poor quality and weak safety awareness of personnel } R_{6} \\
\text { Lack of relevant safety education or training } R_{20}\end{array}$ & $\begin{array}{l}25.80 \\
29.80\end{array}$ & 37.20 \\
\hline \multirow[b]{2}{*}{4} & Nonstandard building materials used in the construction $R_{11}$ & 21.80 & \multirow[b]{2}{*}{55.31} \\
\hline & $\begin{array}{l}\text { Lack of unified, effective management standards or supervision } \\
\text { mechanism } R_{22}\end{array}$ & 49.35 & \\
\hline \multirow[b]{2}{*}{5} & Arbitrary stacking of construction materials $R_{12}$ & 9.30 & \multirow[b]{2}{*}{20.79} \\
\hline & $\begin{array}{c}\text { Mismatch between safety management system and actual construction } \\
\text { conditions } R_{21}\end{array}$ & 14.45 & \\
\hline 6 & $\begin{array}{l}\text { High-altitude operation } R_{1} \\
\text { Improper protection measures for borders and openings } R_{14}\end{array}$ & $\begin{array}{l}25.60 \\
16.80\end{array}$ & 62.64 \\
\hline 7 & $\begin{array}{l}\text { Narrow working surface, insufficient lighting } R_{13} \\
\text { Awful weather } R_{16}\end{array}$ & $\begin{array}{l}12.80 \\
18.25\end{array}$ & 43.59 \\
\hline
\end{tabular}




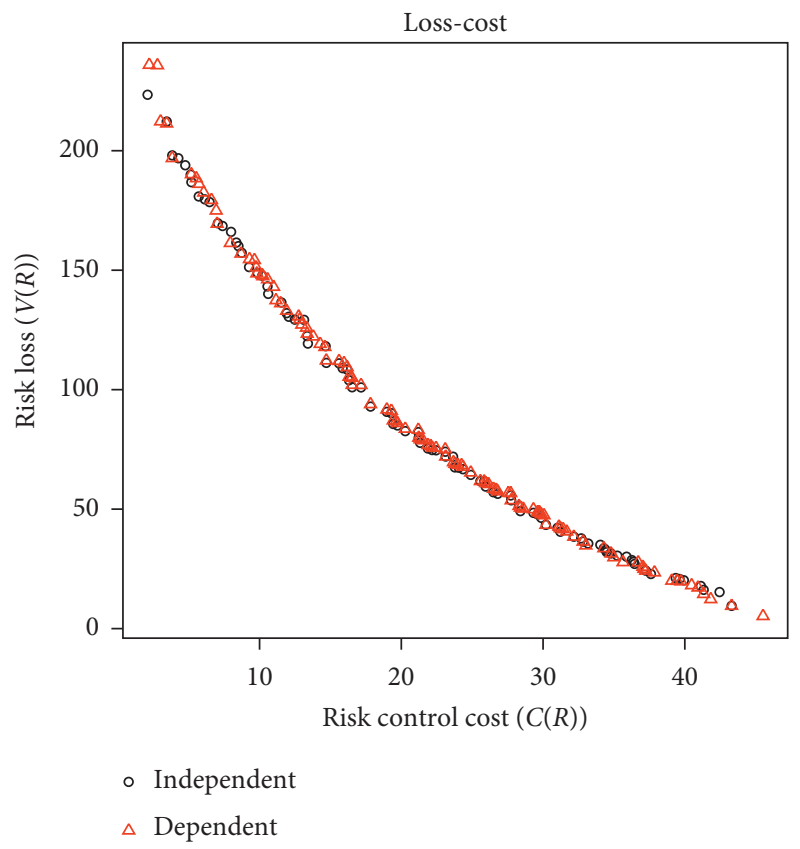

Figure 1: Noninferior solution set under unconstrained conditions.

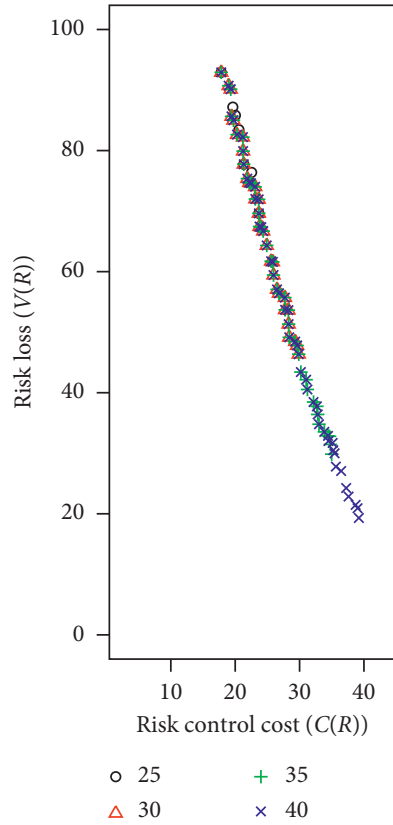

(a)

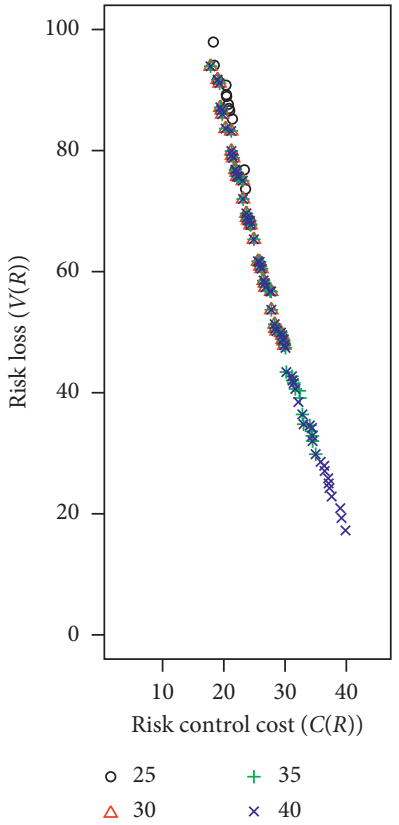

(b)

Figure 2: Noninferior solution sets (a) without considering risk correlations; (b) with considering risk correlations.

are controlled. According to formula (20), the correlation effect no longer exists. Thus, at this time, the results obtained with and without considering correlations differ insignificantly.
The optimal control effects before and after considering risk correlations are comparatively analyzed, and the detailed data are listed in Table 3. Risk managers are recommended to adopt different control strategies based on 

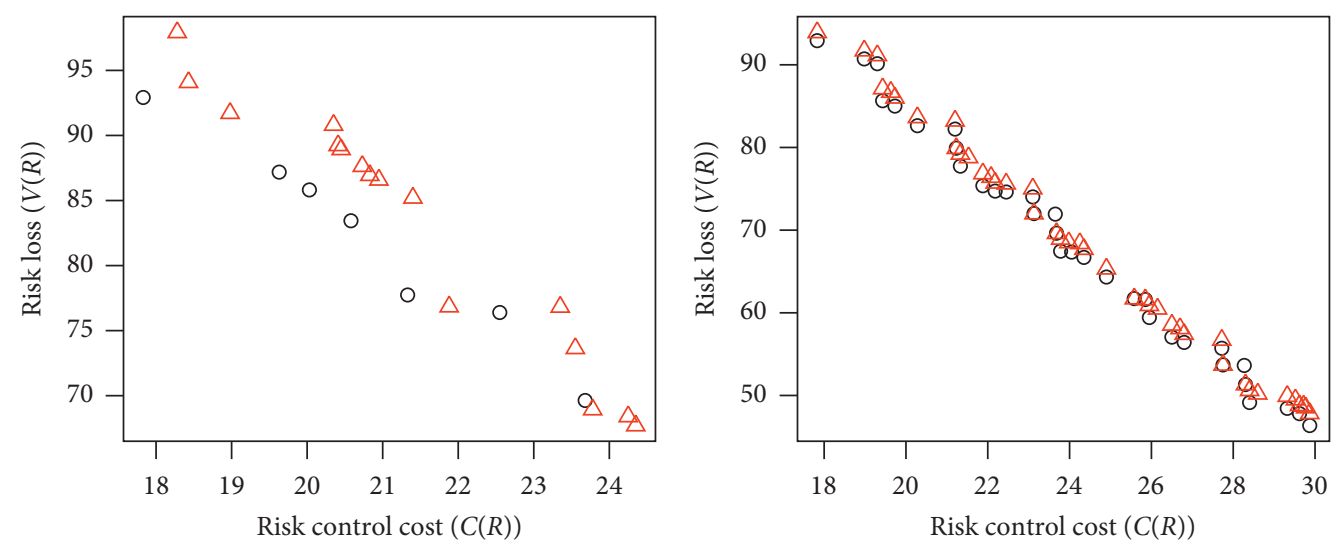

$$
\begin{array}{ll}
\circ \text { Independent } & \circ \text { Independent } \\
\triangle \text { Dependent } & \triangle \text { Dependent }
\end{array}
$$

(a)

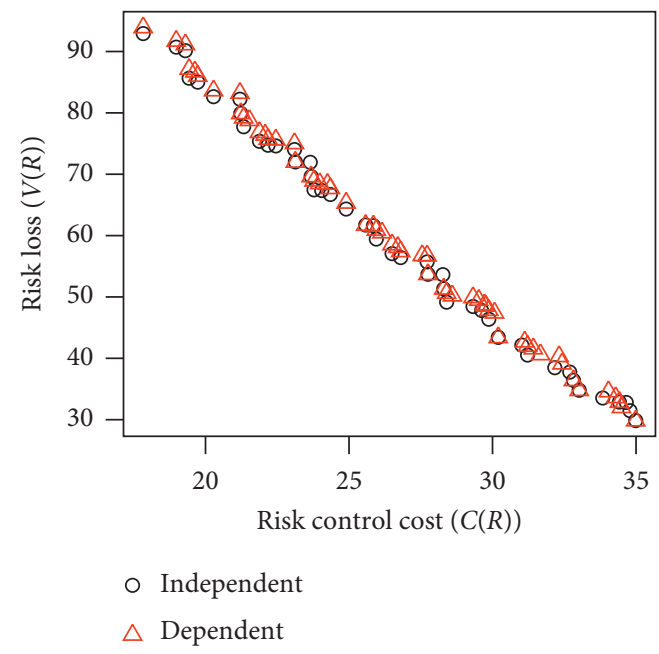

(c)

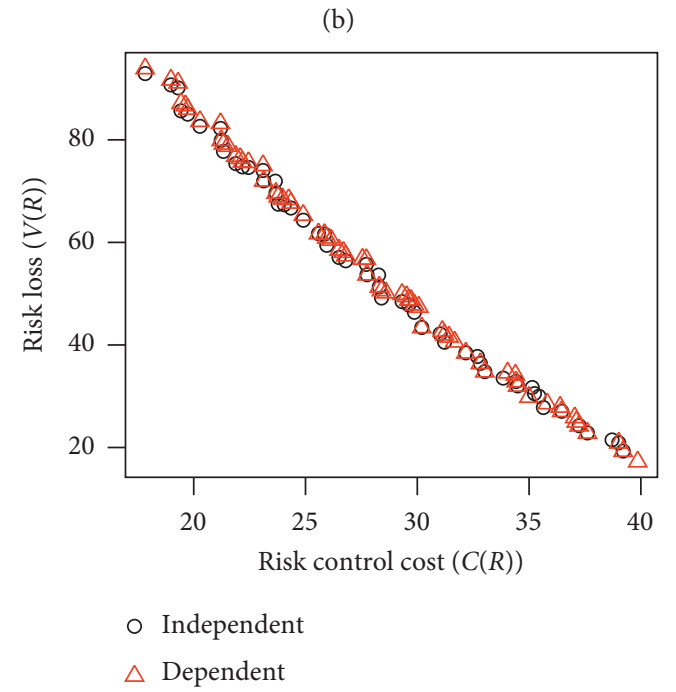

(d)

FIGURE 3: Noninferior solution sets under different constraints: (a) cost constraint: 25; (b) cost constraint: 30; (c) cost constraint: 35; (d) cost constraint: 40 .

the available risk control cost after comprehensively considering a project's safety risk management, as well as risk control cost input and rectification effects. An exemplary analysis is made on Table 3 . The control strategies and global optimal costs under the cost constraints $C(R)$ of 0.3 and 0.35 million yuan are the same. However, the global optimal risk losses differ between scenarios with and without considering risk correlations. Such differences are attributed to the impact of risk correlations. At a cost constraint $C(R)$ of 0.25 million yuan, the global optimal risk loss decreases by 19,200 yuan when the global optimal cost increases by 0.67 million yuan before and after considering the risk correlations. Meanwhile, at a cost constraint $C(R)$ of 0.4 million yuan, the global optimal risk loss decreases by 20,600 yuan when the global optimal cost increases by 0.65 million yuan before and after considering the risk correlations. This suggests that the optimization model can be better rationalized by taking risk correlations into consideration during optimization of risk control cost.

\section{Conclusion}

Safety risk control for prefabricated building construction is an important aspect of building risk management. In this paper, a multiobjective risk control model is created for balancing the system risk level and lowering the cost required for risk control, which is then solved using a discreteMOPSO algorithm. Moreover, the impact of risk correlations is considered into the proposed model for prefabricated building risk control so that it is more in line with the actual situation. However, the impacts among risk factors of prefabricated construction vary. This paper shows 
TABle 3: Optimal control effects under different constraints before and after considering risk correlations.

\begin{tabular}{|c|c|c|c|c|c|c|c|c|}
\hline \multirow{2}{*}{$\begin{array}{l}\text { Constraint } \\
\text { right-hand } \\
\text { parameter } \\
\text { Index }\end{array}$} & \multicolumn{2}{|c|}{0.25 million yuan } & \multicolumn{2}{|c|}{0.3 million yuan } & \multicolumn{2}{|c|}{0.35 million yuan } & \multicolumn{2}{|c|}{0.4 million yuan } \\
\hline & $\begin{array}{l}\text { Without } \\
\text { considering } \\
\text { correlation }\end{array}$ & $\begin{array}{c}\text { Considering } \\
\text { correlation }\end{array}$ & $\begin{array}{l}\text { Without } \\
\text { considering } \\
\text { correlation }\end{array}$ & $\begin{array}{l}\text { Considering } \\
\text { correlation }\end{array}$ & $\begin{array}{l}\text { Without } \\
\text { considering } \\
\text { correlation }\end{array}$ & $\begin{array}{c}\text { Considering } \\
\text { correlation }\end{array}$ & $\begin{array}{l}\text { Without } \\
\text { considering } \\
\text { correlation }\end{array}$ & $\begin{array}{c}\text { Considering } \\
\text { correlation }\end{array}$ \\
\hline$R_{1}$ & 1 & 1 & 1 & 1 & 1 & 1 & 1 & 1 \\
\hline$R_{2}$ & 1 & 1 & 1 & 1 & 1 & 1 & 1 & 1 \\
\hline$R_{3}$ & 0 & 0 & 0 & 0 & 0 & 0 & 0 & 0 \\
\hline$R_{4}$ & 0 & 1 & 1 & 1 & 1 & 1 & 1 & 1 \\
\hline$R_{5}$ & 1 & 1 & 1 & 1 & 1 & 1 & 1 & 1 \\
\hline$R_{6}$ & 1 & 1 & 1 & 1 & 1 & 1 & 1 & 1 \\
\hline$R_{7}$ & 1 & 1 & 1 & 1 & 1 & 1 & 1 & 1 \\
\hline$R_{8}$ & 0 & 0 & 0 & 0 & 0 & 0 & 0 & 0 \\
\hline$R_{9}$ & 0 & 0 & 0 & 0 & 1 & 1 & 1 & 1 \\
\hline$R_{10}$ & 0 & 1 & 1 & 1 & 1 & 1 & 1 & 1 \\
\hline$R_{11}$ & 0 & 0 & 0 & 0 & 0 & 0 & 1 & 1 \\
\hline$R_{12}$ & 0 & 0 & 0 & 0 & 0 & 0 & 0 & 0 \\
\hline$R_{13}$ & 0 & 0 & 0 & 0 & 1 & 1 & 1 & 0 \\
\hline$R_{14}$ & 1 & 1 & 1 & 1 & 1 & 1 & 1 & 1 \\
\hline$R_{15}$ & 1 & 0 & 1 & 1 & 1 & 1 & 1 & 1 \\
\hline$R_{16}$ & 1 & 0 & 0 & 0 & 1 & 1 & 1 & 1 \\
\hline$R_{17}$ & 0 & 0 & 0 & 0 & 0 & 0 & 0 & 1 \\
\hline$R_{18}$ & 0 & 0 & 1 & 1 & 0 & 0 & 0 & 0 \\
\hline$R_{19}$ & 1 & 1 & 1 & 1 & 1 & 1 & 1 & 1 \\
\hline$R_{20}$ & 1 & 1 & 1 & 1 & 1 & 1 & 1 & 1 \\
\hline$R_{21}$ & 1 & 0 & 1 & 1 & 1 & 1 & 1 & 1 \\
\hline$R_{22}$ & 1 & 1 & 1 & 1 & 1 & 1 & 1 & 1 \\
\hline$R_{23}$ & 1 & 1 & 1 & 1 & 1 & 1 & 1 & 1 \\
\hline $\begin{array}{l}\text { Global } \\
\text { optimal risk } \\
\text { loss (10,000 } \\
\text { yuan) }\end{array}$ & 69.63 & 67.71 & 46.37 & 47.84 & 29.85 & 29.86 & 19.31 & 17.25 \\
\hline $\begin{array}{l}\text { Global } \\
\text { optimal } \\
\text { control cost } \\
(10,000 \\
\text { yuan) }\end{array}$ & 23.68 & 24.35 & 29.87 & 29.87 & 34.99 & 34.99 & 39.21 & 39.86 \\
\hline
\end{tabular}

certain limitations since only the risk correlations between two risk factors are considered, while failing to study the correlations among multiple risks in depth. Besides, the control strategies for various risk factors are set as attribute variables, i.e., the 0-1 scheme. In subsequent research, they can be considered as continuous variables. To be specific, different control costs can be input for each factor, thereby taking the degree of control into consideration.

\section{Data Availability}

Previously reported (the risk weights) data were used to support this study and are available at DOI: $10.11717 / \mathrm{j}$.issn. 1673-1387.2018.03.09. These prior studies (and datasets) are cited at relevant places within the text as [22]. The (risk accident rates, risk control costs, and losses from risks) data used to support the findings of this study are included within the article. These data come from a certain $\mathrm{PH}$ assembly building project in China. Due to the protection policy, the project's information cannot be disclosed. The (risk accident rates) data were derived by statistically processing data from similar projects. These prior studies are cited at relevant places within the text as $[8,23]$.

\section{Conflicts of Interest}

The authors declare that they have no conflicts of interest.

\section{Acknowledgments}

This research was supported by the National Natural Science Foundation of China (Project no. 51678375).

\section{References}

[1] H. Liu, "A new perspective of building industrialization to promote rapid development of China's construction," World Construction, vol. 6, no. 2, 2017.

[2] L.-Z. Luo, C. Mao, L.-Y. Shen, and Z.-D. Li, "Risk factors affecting practitioners' attitudes toward the implementation of an industrialized building system," Engineering, Construction and Architectural Management, vol. 22, no. 6, pp. 622-643, 2015. 
[3] C. A. Coello Coello and M. S. Lechuga, "MOPSO: a proposal for multiple objective particle swarm optimization," in Proceedings of the 2002 Congress on Evolutionary Computation, IEEE, Honolulu, HI, USA, pp. 1051-1056, 2002.

[4] A. Elgammal and M. El-Naggar, "Energy management in smart grids for the integration of hybrid wind-PV-FC-battery renewable energy resources using multi-objective particle swarm optimisation (MOPSO)," The Journal of Engineering, vol. 2018, no. 11, pp. 1806-1816, 2018.

[5] H. Han, W. Lu, L. Zhang, and J. Qiao, "Adaptive gradient multiobjective particle swarm optimization," IEEE Transactions on Cybernetics, vol. 48, no. 11, pp. 3067-3079, 2018.

[6] C. Z. Li, J. Hong, F. Xue, G. Q. Shen, X. Xu, and M. K. Mok, "Schedule risks in prefabrication housing production in Hong Kong: a social network analysis," Journal of Cleaner Production, vol. 134, pp. 482-494, 2016.

[7] T. Wang, S. Gao, X. Li, and X. Ning, "A meta-network-based risk evaluation and control method for industrialized building construction projects," Journal of Cleaner Production, vol. 205, pp. 552-564, 2018.

[8] C. Z. Li, G. Q. Shen, X. Xu, F. Xue, L. Sommer, and L. Luo, "Schedule risk modeling in prefabrication housing production," Journal of Cleaner Production, vol. 153, pp. 692-706, 2017.

[9] V. Tavakolan and H. Etemadinia, "Fuzzy weighted interpretive structural modeling: improved method for identification of risk interactions in construction projects," Journal of Construction Engineering and Management, vol. 143, no. 11, 2017.

[10] Y.-W. Kim, S.-H. Han, J.-S. Yi, and S. Chang, "Supply chain cost model for prefabricated building material based on timedriven activity-based costing," Canadian Journal of Civil Engineering, vol. 43, no. 4, pp. 287-293, 2016.

[11] A. Mehrdad, K. Vineet, B. Yu et al., "Optimization modeling of multi-skilled resources in prefabrication: theorizing cost analysis of process integration in off-site construction," $\mathrm{Au}$ tomation in Construction, vol. 95, pp. 1-9, 2018.

[12] H. Xue, S. Zhang, Y. Su, and Z. Wu, "Capital cost optimization for prefabrication: a factor Analysis evaluation model," Sustainability, vol. 10, no. 1, p. 159, 2018.

[13] H. Yang, J. K. H. Chung, Y. Chen, Y. Pan, Z. Mei, and X. Sun, "Ordering strategy analysis of prefabricated component manufacturer in construction supply chain," Mathematical Problems in Engineering, vol. 2018, Article ID 4062871, 16 pages, 2018.

[14] Y. Wu, S. Geng, H. Xu, and H. Zhang, "Study of decision framework of wind farm project plan selection under intuitionistic fuzzy set and fuzzy measure environment," Energy Conversion and Management, vol. 87, pp. 274-284, 2014.

[15] D. Denneberg, Non-Additive Measure and Integral, Kluwer, Dordrecht, Netherlands, 1994.

[16] E. Pap, Null-Additive Set Functions, Kluwer, Dordrecht, Netherlands, 1995.

[17] C. Bao, D. Wu, and J. Li, "A knowledge-based risk measure from the fuzzy multi-criteria decision-making perspective," IEEE Transactions on Fuzzy Systems, vol. 27, no. 5, pp. 1126-1138, 2018.

[18] J. Li, X. Yao, X. Sun, and D. Wu, "Determining the fuzzy measures in multiple criteria decision aiding from the tolerance perspective," European Journal of Operational Research, vol. 264, no. 2, pp. 428-439, 2018.

[19] B. Mayag, M. Grabisch, and C. Labreuche, "A characterization of the 2-additive Choquet integral through cardinal information," Fuzzy Sets and Systems, vol. 184, no. 1, pp. 84-105, 2011.
[20] M. Grabisch, J. L. Marichal, R. Mesiar, and E. Pap, Aggregation Functions, Cambridge University Press, Cambridge, UK, 2009.

[21] Y. Shi and R. Eberhart, "A modified particle swarm optimizer," in Proceedings of the 1998 IEEE International Conference on Evolutionary Computation Proceedings: IEEE World Congress on Computational Intelligence, pp. 69-73, Anchorage, AK, USA, May 1998.

[22] C. Chang, Xi Wu, and Z. Li, "Safety risk assessment of prefabricated building construction based on G1-matter element analysis," Journal of Shenyang Jianzhu University (Social Science Edition), vol. 20, no. 3, pp. 268-274, 2018.

[23] H. Xue, S. Zhang, Y. Su, and Z. Wu, "Factors affecting the capital cost of prefabrication: a case study of China," Sustainability, vol. 9, no. 9, p. 1512, 2017.

[24] Y. Chen, L. Li, J. Xiao, Y. Yang, J. Liang, and T. Li, "Particle swarm optimizer with crossover operation," Engineering Applications of Artificial Intelligence, vol. 70, pp. 159-169, 2018.

[25] H. Mofid, H. Jazayeri-Rad, M. Shahbazian, and A. Fetanat, "Enhancing the performance of a parallel nitrogen expansion liquefaction process (NELP) using the multi-objective particle swarm optimization (MOPSO) algorithm," Energy, vol. 172, pp. 286-303, 2019. 